\title{
Factor de crecimiento epidermal y proteínas totales en saliva de fumadores y no fumadores
}

\section{Epidermal growth factor and total protein in saliva of smokers and non smokers}

\author{
Gutiérrez Nova P*, Olivares Navarrete R**, Leyva Huerta ER***
}

\section{RESUMEN}

La función del Factor de Crecimiento Epidermal (EGF) es mantener la integridad de los tejidos, su receptor se localiza en superficies celulares y se expresa principalmente en epitelios. Nuestro objetivo fue determinar la asociación entre el tabaquismo crónico, el pH, las proteínas totales (PT) y el EGF en saliva de fumadores, Se seleccionaron pacientes masculinos, entre 20 y 60 años, de la clínica de Registro del Postgrado de Odontología de la Universidad. Se tomaron muestras de saliva total estimulada (STHe) y no estimulada (STH) a un grupo de fumadores y no fumadores. Se midió el $\mathrm{pH}$, se cuantificaron las PT por el método de Bradford y para el EGF la técnica de ELISA (ELISA Kit KHG0061 biosource). Los resultados fueron: el pH se encontró ligeramente alcalino en STHe en ambos grupos, la cantidad de PT fue de 1,11 $\pm 0,71$ en no fumadores y 0,87 $\pm 0,31$ $\mathrm{mg} / \mathrm{mL}$ en STH de fumadores. En STHe también existió un decremento en la cantidad de PT de 0,94 \pm 0,47 $0,66 \pm 0,21 \mathrm{mg} / \mathrm{mL}$. El EGF fue en STH de no fumadores 14,8 \pm 5,62 comparado con los fumadores con un promedio de 11,4 $\pm 5,45$. En STHe, el promedio del mismo factor fue en los no fumadores de 7,8 $\pm 5,60 \mathrm{y}$ en los fumadores a $9,1 \pm 6,53 \mathrm{ng} / \mathrm{mL}$. Con una con una confiabilidad del $90 \%$.

Palabras clave: Proteínas totales, factor de crecimiento epidermal, saliva, tabaquismo.

\section{SUMMARY}

The role of Epidermal Growth Factor (EGF) is to maintain tissue's integrity. It's receptor is located on cell surfaces and mainly expressed in epithelia. The aim of this study was to determine the association between chronicle tabaquism, pH, total proteins (TP) and EGF in smoker's saliva. There were selected male volunteers between 20 and 60 years old from the Registration Clinic of the Postgrade Division of UNAM's Dentistry School. There were taken stimulated (sWHS) and not stimulated whole saliva (WHS) samples from groups of smoker's and non smokers. Total proteins (Bradford method), pH and EGF (ELISA Kit KHG0061 biosource) were measured. Results: Slightly alkaline $\mathrm{pH}$ in sWHS in both groups. TP were $1.11 \pm 0.71$ for non smokers WHS and $0.87 \pm 0.31 \mathrm{mg} /$ $\mathrm{mL}$ for smokers WHS. In non smokers WHS EGF were $14.8 \pm 5.62$ compared to smokers WHS which showed 11.4 \pm 5.45 . For sWHS the values were $7.8 \pm 5.60$ in non smokers group and $9.10 \pm 6.53 \mathrm{ng} / \mathrm{mL}$ in smokers group.

Key words: Total proteins, epidermal growth hactor, saliva, smokers.

Fecha de recepción: 23 de abril de 2008.

Aceptado para publicación: 14 de mayo de 2008.

* Laboratorio de Patología Bucal.

** Laboratorio de Biología Celular y Molecular.

División de Estudios de Postgrado e Investigación. Facultad de Odontología. Universidad Nacional Autónoma de México. México.

Gutiérrez Nova P, Olivares Navarrete R, Leyva Huerta ER. Factor de crecimiento epidermal y proteínas totales en saliva de fumadores y no fumadores. Av. Odontoestomatol 2008; 24 (6): 377-383. 


\section{INTRODUCCIÓN}

La Organización Mundial de la Salud (OMS) señala que existen casi 1,100 millones de fumadores en el mundo. Se estima que para el año 2020 habrá más de 8,4 millones de muertes anuales por enfermedades atribuibles al consumo del tabaco (1). En México, el tabaquismo es la principal causa de muerte prevenible y está relacionado con 45 mil fallecimientos cada año (2, 3). El $28 \%$ de la población entre 12 y 65 años de edad del área urbana son fumadores activos, lo que representa más de 13 millones de personas fumadoras. Además, $53 \%$ de la población son fumadores pasivos. El humo del cigarrillo está clasificado como carcinógeno clase A por la OMS y se encuentra asociado a enfermedades como: cáncer, enfisema pulmonar y cardiopatía coronaria en adultos. Cuando se combina el consumo de tabaco con alcohol, el riesgo de padecer cáncer bucal aumenta 15 veces más que en personas que no lo consumen $(4,5)$. Así mismo, el tabaquismo está relacionado con el desarrollo de otras lesiones de la cavidad bucal como la melanosis del fumador, queratosis del fumador y estomatitis nicotínica $(4,6)$. El $\mathrm{pH}$ normal de la saliva varía entre 6 y 7 y está directamente relacionado con los niveles de $\mathrm{CO}_{2}$ en sangre y saliva (7). La concentración de proteínas totales y el flujo salival están sujetos a cambios influenciados por los ritmos circadianos, factores hormonales y psicológicos (8) por el cepillado dental (9) yel ejercicio habitual, ya que el flujo salival disminuye durante la ejercitación aeróbica (10). La concentración total de proteínas es mayor cuando existen infecciones bucales $(11,12)$. La secreción salival está regulada por un control nervioso, en el núcleo salival superior en el puente encefálico y es estimulada por impresiones olfatorias, gustativas y mecánicas $(7,12)$. Factores como el dolor, ciertos medicamentos, enfermedades locales y sistémicas, trastornos psiquiátricos así como la época del año pueden alterar la secreción $(12,13,14)$, Los cambios y/o deficiencias nutricionales también alteran el flujo y composición salival. Los ritmos circadianos afectan no sólo la cantidad del flujo sino también la concentración de componentes como proteínas y electrolitos $(13,15)$.

El Factor de Crecimiento Epidermal (EGF), consiste en una cadena polipeptídica de 53 residuos aminoá- cidos que contiene 3 puentes disulfuro intermoleculares (16). Su función es inducir una serie de respuestas biológicas que son mediadas por su unión específica a un receptor tirosina-cinasa de EGF, localizado en la superficie celular $(15,17)$. El EGF promueve la proliferación celular, regula la diferenciación, modula la organogénesis, promueve la angiogénesis y acelera la cicatrización de las heridas; actúa como un inhibidor de la secreción de ácido gástrico, estimula la proliferación de células embrionarias e incrementa la liberación de calcio del hueso. También promueve la reabsorción ósea y es un potente agente quimiotáctico para fibroblastos y células epiteliales $(15,17)$. El EGF se sintetiza inicialmente como una proteína precursora de 160$170 \mathrm{kDa}$. La forma madura y soluble de EGF es una proteína de 6,4 kDa que es detectable en muchos tejidos y prácticamente todos los fluidos humanos, por ejemplo: orina $(80 \mathrm{ng} / \mathrm{mL})$, jugos gástricos $(0,3 \mathrm{ng} / \mathrm{mL})$, fluidos seminales $(25 \mathrm{ng} / \mathrm{mL})$, sudor axilar (1 ng/mL), líquido amniótico de tercer trimestre de embarazo ( $3 \mathrm{ng} / \mathrm{mL})$ y leche materna (50 ng/mL) $(13,15)$.

La síntesis del EGF en saliva se realiza en las células de los conductos excretores de las glándulas salivales mayores, principalmente en la parótida (18, 19). En la saliva se encuentran dos formas de EGF, $\alpha$ y $\beta$ que forman un dímero potencial, pero predomina la forma biológicamente activa $\beta$ de bajo peso molecular $(6,4 \mathrm{kDa})$. La forma de alto peso molecular, consiste en una porción del precursor de la molécula y lleva dentro de su estructura la forma activa de bajo peso molecular (20). Los niveles de EGF en saliva son más elevados por la mañana y en ayunas y en las comidas disminuye drásticamente su concentración entre un 54 a un $70 \%$. Algunos estudios manejan que los niveles de EGF aumentan con la edad según el desarrollo de las glándulas hasta alcanzar la madurez (21). Otros son contradictorios cuando intentan establecer la concentración de EGF en saliva y su relación con el tabaquismo. En la saliva, el EGF estimula el incremento en el grosor de la mucosa y funciona como un péptido de vigilancia ubicando y promoviendo la reparación de las zonas de mucosa dañada (22). El EGF dispara una cascada de señalización intracelular a través de su unión al receptor de EGF, conocido como ErbB1 (15, 17). 
El objetivo de este trabajo fue analizar la relación entre el hábito del tabaquismo crónico, los niveles de proteínas totales y la concentración del EGF.

\section{MATERIAL Y MÉTODOS}

Se seleccionaron 19 pacientes hombres entre 20 y 60 años, que acudieron la clínica de Registro y Diagnóstico de la División de Estudios de Postrado e Investigación de la Facultad de Odontología de la UNAM en la Ciudad de México que no presentaron lesiones clínicamente visibles en la mucosa bucal y no se encontraban bajo tratamiento médico, se realizó encuesta de historia de tabaquismo y exploración clínica bucal previo consentimiento informado, se tomó muestra de saliva entre las 8:30 y las 11:30 de la mañana, explicándoles que debían abstenerse de comer, fumar y lavarse los dientes dos horas antes.

\section{Recolección de saliva}

Las muestras de saliva se colectaron en un tubo de propileno desechable estéril, con tapa roscable de $15 \mathrm{~mL}$, marca Costar. Un tubo por sujeto para STH y otro para STHe.

\section{Saliva total no estimulada (STH)}

Se indicó al paciente que se enjuagara la boca únicamente con agua, indicándole que colectara la saliva dentro de la boca, con los labios cerrados durante un minuto y la vertiera en el tubo colector. Se repitió la operación dos veces con un minuto de descanso. Se colectó saliva por tres minutos.

\section{Saliva total estimulada (STHe)}

Se le indicó al mismo paciente que se enjuagara la boca nuevamente. Se le dio una porción de $10 \mathrm{~mm}$ de tubo de plástico suave como estímulo masticatorio, indicándole realizar 20 movimientos de masticación por minuto. Al cabo de cada minuto, colocó la saliva secretada en el tubo colector y repitió la operación con un $1 \mathrm{~min}$. de descanso repitiendo la operación 2 veces más.
Los tubos con la saliva se etiquetaron y congelaron a $-70^{\circ} \mathrm{C}$ para su almacenamiento durante la fase de recolección, para el análisis de las muestras se colocaron a $5^{\circ} \mathrm{C}$ durante 24 horas y posteriormente se descongelaron a temperatura ambiente por 2 horas (23).

\section{Determinación de pH}

Se usó un potenciómetro Conductronic modelo 120, con pantalla digital a una resolución de $0,001 \mathrm{pH}$ y $1 \mathrm{mV}$ en rangos de 0,00 a 14,00 unidades de $\mathrm{pH}$ y de 0 a $\pm 1.999 \mathrm{mV}$, cada lectura se realizó por triplicado y se promediaron los tres datos para obtener el valor final.

\section{Cuantificación de proteínas totales}

Se llevó a cabo con la técnica de Bradford con un espectrofotómetro Gene Quant Pro usando una curva estándar de BSA. Cada ensayo se hizo por triplicado y se promediaron las tres lecturas obtenidas en cada caso para obtener el valor final.

\section{Cuantificación de EGF}

La cuantificación de EGF se hizo con la técnica de ELISA empleando un kit comercial para la cuantificación de EGF humano (ELISA Kit KHG0061 biosource). Se midió la absorbancia a $450 \mathrm{~nm}$.

\section{Análisis estadístico}

Se obtuvo el promedio y desviación estándar de las mediciones por triplicado en cada categoría de cada grupo. El análisis de los datos se hizo con U de Mann Whitney.

\section{RESULTADOS}

El grupo consistió en 19 pacientes; 10 no fumadores y 9 fumadores. Se obtuvieron dos resultados para cada paciente en cada medición, uno para STH y otro para STHe del pH, PT y EGF. 
En el grupo de no fumadores encontramos que el pH fue más alcalino en STH que en STHe en 8 casos y ácido en 2 (en STH de 7,07 a 7,86 y en STHe de 7,24 a 8,43). La cantidad de PT fue menor en la mayoría de los casos en STHe que en STH excepto en 3 (en STH de 0,21 a 2,19 mg/mL y en STHe de 0,48 a $1,72 \mathrm{mg} / \mathrm{mL}$ ). La concentración de EGF fue menor en STHe que en STH excepto en un caso (en STH variaron de 7,77 a $22,39 \mathrm{ng} / \mathrm{mL}$ y en STHe de 1,81 a $19,57 \mathrm{ng} / \mathrm{mL}$ ) (Tabla 1).

En el grupo de pacientes fumadores. El pH fue más alcalino en STHe en 6 de los 9 casos y ligeramente ácido en 3, (en STH de 6,5 a 7,85 y en STHe de 7,24 a 8,44). La cantidad de PT fue menor en STH que en STHe en 7 de los 9 casos, (de 0,49 a 1,18 mg/mL en $\mathrm{STH}$ y en STHe de 0,24 a $0,86 \mathrm{mg} / \mathrm{mL}$ ). Con respecto a la concentración de EGF, fue menor en STHe que en STH también en 7 casos y en 2 casos los niveles más altos se encontraron en STHe (en STH variaron de 5,03 a 19,22 ng/mL y en STHe de 2,49 a 22,84 ng/ $\mathrm{mL}$ ) (Tabla 2). En promedio en ambos grupos el pH, fue más alcalino en STHe que en STH, se encontró una menor concentración de PT en STHe que en STH en los dos grupos. Así mismo la concentración de EGF fue menor en STHe que en STH (Tabla 3).

\begin{tabular}{|rrrrrrrr|r|}
\hline \multicolumn{6}{|c|}{$\begin{array}{c}\text { TABLA 1.- NIVELES DE PH, CUANTIFICACIÓN DE PROTEÍNAS TOTALES Y FACTOR DE } \\
\text { CRECIMIENTO EPIDERMAL EN LA SALIVA DEL GRUPO DE NO FUMADORES }\end{array}$} \\
\hline Caso & Edad & pH/STH & pH/STHe & $\begin{array}{l}\text { PT/STH } \\
\text { mg/mL }\end{array}$ & $\begin{array}{c}\text { PT/STHe } \\
\mathbf{m g} / \mathrm{mL}\end{array}$ & $\begin{array}{c}\text { EGF/STH } \\
\text { ng/mL }\end{array}$ & $\begin{array}{r}\text { EGF/STHe } \\
\text { ng/mL }\end{array}$ \\
\hline 1 & 21 & 7,59 & 7,7 & 0,21 & 0,48 & 13,99 & 1,81 \\
2 & 22 & 7,29 & 7,24 & 2,19 & 1,72 & 22,39 & 19,57 \\
3 & 25 & 7,73 & 7,68 & 0,82 & 0,59 & 10,46 & 6,71 \\
4 & 27 & 7,86 & 7,93 & 1,99 & 1,50 & 14,54 & 12,18 \\
5 & 30 & 7,07 & 7,77 & 0,88 & 0,49 & 22,45 & 2,93 \\
6 & 33 & 7,34 & 8,04 & 0,58 & 0,68 & 7,77 & 9,91 \\
7 & 42 & 7,59 & 7,98 & 0,85 & 0,72 & 9,36 & 1,95 \\
8 & 49 & 7,18 & 7,66 & 1,00 & 1,30 & 12,26 & 6,78 \\
9 & 51 & 7,78 & 8,43 & 0,78 & 0,60 & 12,09 & 4,51 \\
10 & 54 & 7,28 & 7,6 & 2,03 & 1,31 & 22,28 & 11,46 \\
\hline
\end{tabular}

TABLA 2.- PACIENTES FUMADORES NIVELES DE PH, CUANTIFICACIÓN DE PROTEÍNAS TOTALES Y FACTOR DE CRECIMIENTO EPIDERMAL EN LA SALIVA DEL GRUPO DE FUMADORES

\begin{tabular}{|llllccrr|}
\hline Caso & Edad & pH/STH & pH/STHe & $\begin{array}{c}\mathrm{PT} / \mathrm{STH} \\
\mathrm{mg} / \mathrm{mL}\end{array}$ & $\begin{array}{c}\mathrm{PT} / \mathrm{STHe} \\
\mathrm{mg} / \mathrm{mL}\end{array}$ & $\begin{array}{c}\mathrm{EGF} / \mathrm{STH} \\
\mathrm{ng} / \mathrm{mL}\end{array}$ & $\begin{array}{r}\mathrm{EGF} / \mathrm{STHe} \\
\mathrm{ng} / \mathrm{mL}\end{array}$ \\
\hline 11 & 22 & 7,28 & 7,9 & 1,18 & 0,86 & 15,13 & 8,53 \\
12 & 23 & 7,85 & 7,77 & 0,77 & 0,80 & 5,03 & 2,55 \\
13 & 23 & 7,3 & 8,44 & 1,35 & 0,71 & 14,8 & 3,88 \\
14 & 29 & 7,36 & 7,22 & 0,58 & 0,61 & 7,97 & 11,67 \\
15 & 33 & 7,39 & 7,64 & 0,49 & 0,24 & 7,96 & 22,84 \\
16 & 35 & 7,57 & 7,44 & 1,02 & 0,55 & 18,04 & 5,94 \\
17 & 42 & 6,93 & 6,95 & 0,77 & 0,51 & 19,22 & 11,22 \\
18 & 49 & 6,5 & 8,09 & 1,13 & 0,84 & 9,8 & 2,49 \\
19 & 60 & 6,76 & 7,19 & 0,58 & 0,84 & 5,04 & 13,2 \\
\hline
\end{tabular}




\begin{tabular}{|c|c|c|c|c|c|c|}
\hline \multicolumn{7}{|c|}{$\begin{array}{c}\text { TABLA 3.- PROMEDIO Y DESVIACIÓN ESTÁNDAR DEL PH, PROTEÍNAS TOTALES Y EGF } \\
\text { EN SALIVA TOTAL ESTIMULADA Y NO DE AMBOS GRUPOS }\end{array}$} \\
\hline Grupo & $\mathrm{pH} / \mathrm{STH}$ & $\mathrm{pH} / \mathrm{STHe}$ & $\begin{array}{l}\mathrm{PT} / \mathrm{STH} \\
\mathrm{mg} / \mathrm{mL}\end{array}$ & $\begin{array}{l}\mathrm{PT} / \mathrm{STHe} \\
\mathrm{mg} / \mathrm{mL}\end{array}$ & $\begin{array}{l}\mathrm{EGF} / \mathrm{STH} \\
\mathrm{ng} / \mathrm{mL}\end{array}$ & $\begin{array}{l}\mathrm{EGF} / \mathrm{STHe} \\
\mathrm{ng} / \mathrm{mL}\end{array}$ \\
\hline No fumadores & $7,5 \pm 0,27$ & $7,8 \pm 0,3$ & $1,11 \pm 0,71$ & $0,94 \pm 0,47$ & & $7,8 \pm 5,60$ \\
\hline Fumadores & $7,2 \pm 0,42$ & $7,6 \pm 0,48$ & $0,87 \pm 0,31$ & $0,66 \pm 0,21$ & $11,4 \pm 5,45$ & $9,1 \pm 6,53$ \\
\hline
\end{tabular}

En el análisis estadístico, se encontró que la cantidad de EGF en STH de pacientes fumadores fue significativamente menor a la encontrada en STH de pacientes no fumadores, a un $90 \%$ de confiabilidad. El pH de STH fue significativamente mayor al de STHe en ambos grupos (no fumadores y fumadores) al 95\% de confiabilidad. No hubo diferencia significativa al comparar los valores de $\mathrm{pH}$ entre ambos grupos.

\section{DISCUSIÓN}

Grigoriev, y cols., en 2003, encontraron que los estados depresivos iban acompañados de una disminución en la cantidad de proteínas en los rangos de 20 a 200 kD y en cambio, estados de ánimo positivos provocaban el efecto contrario (8). Nagler, R. y cols. (24), analizaron los efectos e interacciones de la fase gaseosa del humo de cigarro en contacto con la cavidad bucal, reportando que el humo del cigarro produce un aumento inmediato en la expresión de los carbonilos proteicos donde el humo del cigarro indujo pérdida de propiedades enzimáticas salivales. Estos datos muestran una relación entre la concentración y función de determinados tipos proteicos y el consumo del tabaco. Otros estudios reportan diferentes cantidades de EGF en saliva de pacientes sanos no fumadores. Thesleff y cols. (19), midieron la concentración de EGF en STH; en saliva parotídea y combinada de las glándulas sublingual y submandibular, encontrando que la concentración normal de EGF en STH fue de 0,864 ng/mL, en saliva de glándula parótida 2,704 ng/mL y en saliva de glándulas sublingual y submandibular 0,357 ng/mL. Motoko, I. y cols. (16), midieron los niveles de EGF en saliva, orina y suero y la expresión inmunohistoquímica de EGF en glándulas salivales normales, encontraron que la concentración de EGF variaba dependiendo de la edad: de 0 a 9 años 3,06 \pm 0,32 $\mathrm{ng} / \mathrm{mL}$ y en personas mayores de 10 años era de 4,7 $\pm 3,5 \mathrm{ng} / \mathrm{mL}$. Los niveles de EGF fueron de 5,7 \pm $4,03 \mathrm{ng} / \mathrm{mL}$ en hombres y 4,36 $\pm 3,00$ en mujeres, pero sin diferencia significativa en ambos grupos; ellos concluyeron que el incremento de EGF con la edad es ligado al desarrollo de las glándulas salivales y se detiene al alcanzar la madurez. Los niveles de EGF son mas elevados en condiciones de ayuno, disminuyendo durante las comidas. Las proporciones relativas de EGF en saliva submandibular, parotídea y STH fueron 1:6:4. Ambos autores coinciden en que la glándula parótida es la principal secretora de EGF, pero en cuanto a los valores que encontraron en ambos casos resultaron mucho menores a los que nosotros encontramos de $14,8 \mathrm{ng} / \mathrm{mL}$ en STH de pacientes sanos no fumadores. Tomando en cuenta los datos de Motoko, realizamos la recolección de muestras entre las 8:30 y las 11:30 de la mañana, en pacientes en ayunas para evitar la influencia de los ritmos circadianos.

Existe discrepancia en cuanto a los valores del EGF en fumadores; Tunio y cols. (25), midieron la cantidad de EGF en saliva y jugo gástrico en condiciones basales y después de la estimulación con histamina en 60 pacientes, 20 voluntarios sanos, 20 pacientes con úlcera duodenal y 20 con dispepsia sin úlceras. No encontraron diferencias significativas entre los tres grupos, tampoco encontraron diferencias en la concentración de EGF por género, o en fumadores y no fumadores. Los valores en saliva de EGF fueron $3,62,4,59$ y $2,68 \mathrm{ng} / \mathrm{mL}$ para pacientes normales, con úlcera y dispepsia respectivamente. De igual manera, Trudgill, N. y cols. (22) encontraron que a pesar de que el tabaquismo está asociado con una menor concentración salival de bicarbonato y menor flujo salival no había diferencias en los niveles de EGF entre fumadores y no fumadores. Manccini y 
cols. (26), no encontraron diferencias significativas en los niveles de EGF en STH entre fumadores y no fumadores al estudiar saliva y jugo gástrico de pacientes con enfermedad péptica. Nosotros encontramos en nuestra muestra que la concentración de EGF fue menor en STHe que en STH y una concentración levemente mayor entre no fumadores al compararla con fumadores.

McGurk, M. y cols. (20), analizaron los niveles de EGF en STH y STHe de voluntarios sanos y concluyeron que están relacionados con el flujo salival, pero con un coeficiente de correlación bajo ( $r=0,47)$. Encontraron ambas formas del EGF (de alto y bajo peso molecular) predominando el de bajo peso. Tras la estimulación durante tres minutos, el EGF de alto peso molecular se incrementa de 18 a 38\%. Ellos consideraron que los ritmos circadianos tienen influencia principalmente en la STH. Jones, y cols. (25), reportaron niveles significativamente menores de EGF en STHe. Mandel refiere que Wang, y cols. (27), encontraron niveles menores de EGF en STH de fumadores crónicos en personas que consumían más de 15 cigarros diarios. Nosotros encontramos menor concentración de EGF en fumadores (11,4 ng/ $\mathrm{mL})$ que en no fumadores $(14,8 \mathrm{ng} / \mathrm{mL})$ en $\mathrm{STH}, \mathrm{Se}$ tomaron como fumadores positivos los pacientes que consumieron mínimo 6 cigarros diarios según parámetros de tabaquismo de la OMS. Konturek, J. y cols. (28), encontraron niveles de EGF salivales significativamente menores en fumadores que en no fumadores al igual que Ma, L. y cols. (29), ellos mencionan que el tabaquismo provoca retardo en la cicatrización de heridas como consecuencia de una reducción en la angiogénesis, proliferación celular y secreción mucosa debido a una depresión en la biosíntesis de EGF y la expresión de su mRNA en glándulas salivales y mucosa gástrica.

\section{CONCLUSIONES}

El pH de STHe es más alcalino que STH en cada grupo. Se encontraron algunos valores ácidos en el grupo de fumadores pero sin significancia estadística. Los pacientes fumadores tuvieron menor concentración de EGF en STH en comparación con pacientes no fumadores y no hubo variación en la concentración de proteínas totales.

\section{BIBLIOGRAFÍA}

1. Majid Ezzati y Alan López, Estimates of Global Mortality Attributable to Smoking in 2000, The Lancet, 2003;362:847-52.

2. Belasso Guido. "El Programa de Acción Contra el Tabaquismo", Salud pública de México 2002; 44(suppl. 1):s5-s6.

3. Encuesta Nacional de Adicciones 1998, Secretaría de Salud, Dirección Nacional de Epidemiología, México, 1999.

4. Shulman JD, Beach MM, Rivera-Hidalgo F. The Prevalence of Oral Mucosal Lesions in U.S. Adults: Data From the Third National Health and Nutrition Examination Survey 1988-1994. J Am Dent Assoc. 2004 sept;135(9):1279-86

5. Tovar-Guzman VJ, Barquera S, López- Antunano FJ. Mortality Trends in Cancer Attribuitable to Tobacco in México. Salud Pública Mex 2002;44 suppl 1:S20-8.

6. K. Andre, S. Shraub, et. al. Role of Alcohol and Tobacco in the Aetiology of Head and Neck Cancer: A Case Control Study in the Doubs Region of France. Oral Oncol. Eur J Cancer 1995; 31B(5):301-9.

7. Sue P. Humphry, MSEd, y Russell T. Williamson, $\mathrm{DMD}^{\mathrm{b}}$, A Review of Saliva: Normal Composition, Flow and Function. J Prosthtet Dent 2001;85: 162-9.

8. Grigoriev et. al. Protein Content of Human Saliva in Various Psycho-emotional States, Biochemistry Moscow 2003;68(4):405-6.

9. Hok, Brand et. al. Toothbrushing Affects the protein Composition of Whole Saliva. Eur J Oral Sci 2002;110:480-1.

10. Chicharro, Lucía, et.al. Saliva Composition and Exercise. Sports medicine 1998; Jul 26-1.

11. Banderas, J. A y González M. Saliva y Cavidad Bucal: Parte II, Proteínas Salivales: Funciones Bio- 
lógicas en el Mantenimiento de la Homeostasis Bucal. Práctica Odontológica 1994;15(7):13-20.

12. C. Dawes y B. Y Ong. Circadian Rhythms in the Flow Rate and Proportional Contribution of Parotid to Whole Saliva Volume in Man. Archs Oral Biol 1973;18:1145-53.

13. Pesonen, Viinikka, et. al. Size Heterogenecy of Epidermal Growth Factor in Human Body Fluids. Life Sciences 1987;40:2489-94.

14. Bardow, Nyvad et. al. Relatonships Between Medication Intake, Complaints of Dry Mouth, Salivary Flow Rate and Composition, and the Rate of Tooth Demineralization in Situ. Archs Oral Biol 2001;46:413-23.

15. Johannes Boonstra, Philip Rijken, Bruno Humbel et. al. The Epidermal Growth Factor. Cell Biology International 1995;19(5).

16. Ino M, Ushiro K, Ino C, Yamashita T, Kumazawa T. Kinetics of Epidermal Growth Factor in Saliva. Acta Otolaryngol (Suppl) 1993;500:126-130.

17. Carpenter, G y Cohen, S. Epidermal Growth Factor. J Biol Chem 1990;265(14):7709-12.

18. Zelles T, Purushotham KR, Macauley SP, Oxford GE, Humphereys-Beher MG. Saliva and Growth Factors: the Fountain of Youth Resides in us All. J Dent Res 1995;74(12):1826-32.

19. Thesleff I, Viinikka L, Saxen L, Lehtonen E, Perheentupa J. The Parotid Gland is the Main Source of Human Salivary Epidermal Growth Factor. Life Sci 1988;43:13-18.

20. M. McGurk, L Hanford, S. Justice y R. A. Metcalfe. The Secretory Characteristics of Epidermal Growth Factor in Human Saliva. Arch Oral Biol 1990;35(8):653-659.

21. Joh T, Itoh M, Matsumi K, Yokoyama Y, Takeuchi T, Kato T, et. al. Physiological Concentrations of Human Epidermal Growth Factor in Biological Fluids: Use of a Sensitive Enzyme Inmunoassay. Clinica Chim Acta 1986; Jul 158 (1):81-90.
22. Trudgill NJ, Smith LF, Kershaw J, Riley SA. Impact of Smoking Cessation on Salivary Function in Health Volunteers. Scand J Gastroenterol 1998;33(6):568-71.

23. Navazesh M, Chirtensen CM. A Comparison of Whole Mouth Resting and Stimulated Salivary Measurement Pocedures. J Dent Res 1982;oct 61(10):1158-62.

24. Nagler R, Lischinsky S, Diamond E, Driques N, Klein I, Reznick AZ. Effect of Cigarette Smoke on Salivary Proteins Enzyme Activities. Arch Biochem Biophis 2000;jul 379(2):229-36.

25. Tunio AM, Hobsley M, Epidermal Growth Factor in Saliva and Gastric Juice: Response to Histamine. Gut 1995; sept 37(3):335-9.

26. Manccini DM, Veit BC. Salivary Epidermal Growth Factor in Patients With and Without Peptic Disease. Am J Gastroenterol 1990;Sep 85 (9):1102-4.

27. Mandel ID. The Functions of Saliva. J Dent Res 1987;feb 66 (Spec Iss): 623-7.

28. Konturek SJ, Bielansky W, Konturek JW, Olesky $\mathrm{J}$, Yamazaki J. Release and Action of EGF on Gastric Secretion in Humans. Scand J Gastroenterol 1989; may 24(4):485-92.

29. Ma L, Wang WP, Chow JY, Yuen ST, Cho CH. Reduction of EGF is Associated With the Delay of Ulcer Healing by Cigarrette Smoking. Am J Physiol Gastrointest Liver Physiol 2000;278:G10-G17.

\section{CORRESPONDENCIA}

Elba Rosa Leyva Huerta

Laboratorio de Patología Clínica y Experimental División de Estudios de Posgrado e Investigación Facultad de Odontología.

Universidad Nacional Autónoma de México Circuito Institutos S/N Ciudad Universitaria. Delegación Coyoacán

CP 04510. México D.F. MEXICO

Tel. 56225560 FAX 55503497

docelbaleyva@hotmail.com 
